\title{
The Complexity of 2-Coloring and Strong Coloring in Uniform Hypergraphs of High Minimum Degree
}

\author{
Edyta Szymańska|j \\ Faculty of Mathematics and Computer Science, Adam Mickiewicz University, Poznań, Poland \\ received $2^{\text {nd }}$ August 2011, revised $18^{\text {th }}$ March 2013, accepted 17th June 2013.
}

In this paper we consider the problem of deciding whether a given $r$-uniform hypergraph $H$ with minimum vertex degree at least $c\left(\begin{array}{c}|V(H)|-1 \\ r-1\end{array}\right)$, or minimum degree of a pair of vertices at least $c\left(\begin{array}{c}|V(H)|-2 \\ r-2\end{array}\right)$, has a vertex 2-coloring. Motivated by an old result of Edwards for graphs, we obtain the first optimal dichotomy results for 2-colorings of $r$-uniform hypergraphs. For each problem and each $r \geq 3$, we determine a threshold value depending on $r$ such that the problem is NP-complete for $c$ below the threshold, while for $c$ strictly above the threshold it is polynomial. We provide an algorithm constructing the coloring with time complexity $O\left(n^{C}\right)$, for some $C=C(c, r)>0$. This algorithm becomes more efficient in cases $r=3,4,5$ due to known Turán numbers of the triangle and the Fano plane. In addition, we determine the computational complexity of strong coloring of 3-uniform hypergraphs $H$ with minimum vertex degree at least $c\left(\begin{array}{c}|V(H)|-1 \\ 2\end{array}\right)$, for some $c$, leaving a gap for $k \geq 5$, which vanishes as $k \rightarrow \infty$.

Keywords: complexity, coloring, hypergraphs

\section{Introduction}

A hypergraph $H=(V, E)$ is a finite set of vertices $V$ together with a family $E$ of distinct, nonempty subsets of vertices called edges. In this paper we consider $r$-uniform hypergraphs $(r$-graphs) in which, for a fixed $r \geq 2$, each edge is of size $r$.

For an $r$-graph $H$, the minimum ( $l$-wise) degree, for $1 \leq l \leq r-1$, denoted by $\delta_{l}(H)$, is the largest integer $d$ such that every $l$-element set of vertices of $H$ is contained in at least $d$ edges of $H$. We will mostly consider the cases $l=1,2$.

A $k$-coloring of a hypergraph $H=(V, E)$ is a function assigning colors from $\{1,2, \ldots, k\}$ to vertices of $H$ in such a way that no edge is monochromatic. The minimum number $k$ such that $H$ admits a $k$ coloring is called the chromatic number of $H$, denoted by $\chi(H)$. A hypergraph with $\chi(H) \leq k$ is called $k$-colorable. A 2-colorable hypergraph is called bipartite.

We study the following decision problem.

${ }^{\dagger}$ Email: edka@amu. edu.pl. Research supported in part by grant N206 017 32/2452 and by grant N206 565740. 
Definition 1.1 For fixed integers $r, 1 \leq l \leq r-1$ and $k$, and a real number $0 \leq c \leq 1$, define the problem $\Pi^{r, l}(k, c)$ as follows:

Input: $r$-graph $H=(V, E)$ with $|V(H)|=n$ and $\delta_{l}(H) \geq c\left(\begin{array}{c}n-l \\ r-l\end{array}\right)$

Output: Is $H$ k-colorable $(\chi(H) \leq k)$ ?

In particular, if we disregard the minimum degree condition by setting $c=0$, we get the classical problem which asks whether a given $r$-graph admits a $k$-coloring. Let us denote this problem by $\Pi^{r}(k)$. For graphs $(r=2)$, it is well known that $\Pi^{2}(2)$ is in $\mathrm{P}$, whereas for every $k \geq 3$ the problem $\Pi^{2}(k)$ is NP-complete [11].

In [8] Edwards considered the problem $\Pi^{2,1}(k, c)$ and proved the following result.

Theorem 1.2 (Th. 2.5, $[\mathbf{8}])$ For $k \geq 3$,

$$
\Pi^{2,1}(k, c) \text { is }\left\{\begin{array}{l}
\text { NP-complete, for } c \leq \frac{k-3}{k-2}, \\
\text { in } P, \text { for } c>\frac{k-3}{k-2} .
\end{array}\right.
$$

Moreover, the time complexity of Edwards's algorithm is

$O\left(\max \left\{n^{k-1} \log n ; n^{\frac{k-2}{\log _{k}(1-\gamma)^{-1}}+1}\right\}\right)$, for some $\gamma=\gamma(c, k)>0$.

We extend this result to $r$-graphs for $r \geq 3$ and $l=1,2$ and determine the threshold values of the constant $c$ above which $\Pi^{r, l}(2, c)$ is in $\mathrm{P}$ and below which $\Pi^{r, l}(2, c)$ is NP-complete. The following theorem is the main result of this paper.

Theorem 1.3 For every $r \geq 3$ and $l=1,2$,

$$
\Pi^{r, l}(2, c) \text { is }\left\{\begin{array}{l}
N P \text {-complete, for } c<1-\frac{1}{2^{r-l-1}}, \\
\text { in } P, \text { for } c>1-\frac{1}{2^{r-l-1}} .
\end{array}\right.
$$

Moreover, in the latter case, the time complexity of $\Pi^{r, l}(2, c)$ is

$O\left(\max \left\{n^{4 / \epsilon+l} \log n ; n^{\frac{4 l}{\log (1-\epsilon / 2)^{-1}}+1}\right\}\right)$ with $\epsilon=c-1+\frac{1}{2^{r-l-1}}$.

On the hardness side of the above result, we show that the problem $\Pi^{r, l}(2, c)$ is NP-complete for $c<1-\frac{1}{2^{r-l-1}}$ for all $1 \leq l<r$. This suggests that the value $1-\frac{1}{2^{r-l-1}}$ is indeed the threshold in general.

Conjecture 1.4 For every $r \geq 3$ and $1 \leq l<r$, the problem $\Pi^{r, l}(2, c)$ is in $P$, for $c>1-\frac{1}{2^{r-l-1}}$.

In addition, for 3-,4- and 5-graphs we apply known results from extremal hypergraph theory and obtain modified algorithms, often with better running time.

Theorem 1.5 The complexity of

a) $\Pi^{3,2}(2, c)$ is $O\left(\max \left\{n^{3} \log n ; n^{\frac{2}{\log (1-c)^{-1}}+1}\right\}\right)$, for $c>0$, 
b) $\Pi^{3,1}(2, c)$ is $O\left(\max \left\{n^{3} \log n ; n^{\frac{3}{\log (1-\epsilon)^{-1}}+1}\right\}\right)$ and of $\Pi^{4,2}(2, c)$ is $O\left(\max \left\{n^{4} \log n ; n^{\frac{3}{\log (1-\epsilon)^{-1}}+1}\right\}\right)$, for $c>\frac{1}{2}$ and $\epsilon=c-\frac{1}{2}$,

c) $\Pi^{4,1}(2, c)$ is $O\left(\max \left\{n^{8} \log n ; n^{\frac{7}{\log \left(1-\epsilon^{8} / 2\right)^{-1}}+1}\right\}\right)$ and of $\Pi^{5,2}(2, c)$ is $O\left(\max \left\{n^{9} \log n ; n^{\frac{7}{\log \left(1-\epsilon^{8} / 2\right)^{-1}+1}}\right\}\right)$, for $c>\frac{3}{4}$ and $\epsilon=c-\frac{3}{4}$.

In the case of hypergraphs yet another version of coloring is also possible. A given $k$-coloring of $H$ is called strong if for every edge of $H$, each color appears at most once in it. Analogously, the minimum number $k$ such that $H$ admits a strong $k$-coloring is called the strong chromatic number of $H$, denoted by $\chi_{s}(H)$. Notice that $\chi(H) \leq \chi_{s}(H)$, and for any $r$-graph $H$, it holds $\chi_{s}(H) \geq r$. The corresponding decision problem is given below.

Definition 1.6 For fixed integers $r, 1 \leq l \leq r-1$ and $k \geq r$, and a real number $0 \leq c \leq 1$, define the problem $\Pi_{s}^{r, l}(k, c)$ as follows:

Input: $r$-graph $H=(V, E)$ with $|V(H)|=n$ and $\delta_{l}(H) \geq c\left(\begin{array}{l}n-l \\ r-l\end{array}\right)$

Output: Is $H$ strong $k$-colorable $\left(\chi_{s}(H) \leq k\right)$ ?

This problem reduces to a graph coloring problem in the following sense.

Remark 1.7 Every strong coloring of an r-graph can be viewed as a vertex coloring of the clique graph $G_{r}(H)$ of the r-graph $H$, defined on the same set of vertices, with edge set $E\left(G_{r}(H)\right)=\{\{u, v\}: u, v \in$ e for some $e \in E(H)\}$. In this way, $\chi_{s}(H)=\chi\left(G_{r}(H)\right)$, the ordinary chromatic number of the clique graph of $H$.

Using this relation together with Theorem 1.2 and complementing it with a proof of NP-completeness, we obtain the following result.

Proposition 1.8 For $k \geq 3, \Pi_{s}^{3,1}(k, c)$ is $\left\{\begin{array}{l}N P \text {-complete, for } c \leq \frac{(k-3)(k-4)}{(k-2)^{2}}, \\ \text { in } P \text {, for } c>\left(\frac{k-3}{k-2}\right)^{2}\end{array}\right.$

For $k=3$ we see that $\Pi_{s}^{3,1}(3, c)$ is in $\mathrm{P}$ for all $c>0$, while for $k \geq 4$ there is a gap between the two thresholds. We close the gap for $k=4$.

Theorem $1.9 \Pi_{s}^{3,1}(4, c)$ is in $P$, for every $c>0$.

Remark 1.10 For $l \geq 2$ the problem $\Pi_{s}^{r, l}(k, c)$ is trivial for any $c>0$. This is because in such a case, the condition $\delta_{l}(H) \geq c\left(\begin{array}{l}n-l \\ k-l\end{array}\right)>0$ implies that every set of l vertices belongs to a hyperedge and thus, every strong coloring is a one-to-one mapping, which imposes $\chi_{s}(H)=n$.

In view of Remark 1.10 , we only consider strong coloring in the case of $l=1$ and use a shorthand notation $\Pi_{s}^{r}(k, c):=\Pi_{s}^{r, 1}(k, c)$.

This paper is organized in the following manner. In the next section we give an overview of previous results. Section 3 contains the proof of the hardness result for any $r \geq 3$ and $1 \leq l<r$. The polynomial time algorithms constructing a 2-coloring in dense $r$-graphs (the proof of Theorem 1.3) as well as in 3-, 4- 
and 5-graphs (the proof of Theorem 1.5 are presented in Section 4. That section also includes a comment on a corresponding enumeration problem. Section 5 concerns the strong coloring problem for 3-graphs. The paper is concluded with some final remarks and open questions.

\section{Known Results}

The property of hypergraph 2-colorability has been studied since the paper of Bernstein [2] and it has got its other name, Property $B$, after him. It is a central problem in combinatorics as well as in computer science due to its connections to graph coloring and satisfiability problems. A line of research (e.g. [23]) has been devoted to extremal problems asking for the least number of edges that an $r$-graph can have without possessing Property B.

From the computer science point of view, one interesting issue is the approximability of this NPcomplete problem. Guruswami et al. [13] proved that it is NP-hard to color bipartite $r$-graphs with a constant number of colors for $r \geq 4$. Dinur et al. [7] showed that this problem remains inapproximable by a constant for 3-graphs. On the other hand, Krivelevich et al. [19] found a polynomial time algorithm which colors bipartite 3-graphs using $O\left(n^{1 / 5} \log ^{c} n\right)$ colors.

Another direction of investigation is to look for an optimal solution in random or restricted classes of $r$-graphs. Recently, an expected polynomial time algorithm for coloring 2-colorable random 3-graphs with two colors was given by Person and Schacht in [21].

The purpose of our paper is to study the complexity of this problem for dense hypergraphs, which was first addressed by Chen and Frieze in [4]. They showed, using the idea of Edwards [8], that every bipartite 3-graph with $\delta_{2}(H)>c n$ can be 2-colored in $n^{O(1 / c)}$ time via a randomized algorithm. Their result, as stated, relies on the assumption that the input hypergraph is, indeed, 2-colorable. Similarly to Edwards, they reduce the problem to 2-SAT, but their 2-SAT formulation is different. We introduce the link graphs into this idea and first show a deterministic algorithm for the same set of parameters $(r=3, l=2)$ with running time $O\left(n^{\frac{2}{\log (1-c)^{-1}}+1}\right)$. Then we extend it to $r$-graphs for every $r \geq 3$. In addition, we consider $\delta_{1}(H)$, i.e. the degree of a single vertex instead of a pair and get similar results for every $r \geq 3$.

At the same time, another motivation to consider the 2-coloring problem in dense hypergraphs came from a recent study of other problems in this class. In the last ten years a series of papers appeared (see, e.g. [26],[25],[22]) where the structural properties of dense hypergraphs, satisfying the so called Diracconditions were studied. They triggered further investigation of the computational aspects of problems like matching, Hamilton cycle and packing. The complexity of perfect and almost perfect matching in hypergraphs of large minimum $(r-1)$-wise degree was examined in [28] and [16]. In [15], the first polynomial time algorithm finding a Hamiltonian cycle in $r$-graphs with minimum degree $\delta_{r-1}(H) \geq$ $(1 / 2+\epsilon) n$ was given.

Strong colorings of general hypergraphs were studied by Agnarsson and Halldórsson in [1]. Their main motivation was to unify various coloring problems as strong colorings of appropriate hypergraphs and their results contain offline and online approximation algorithms for strong colorings of arbitrary hypergraphs in terms of the size of a largest hyperedge and the number of hyperedges.

A preliminary version of this paper appeared in [27]. 


\section{Hardness Result}

In this section we prove the first part of Theorem 1.3 in a more general form.

Lemma 3.1 For every $r \geq 3,1 \leq l<r$ and $c<1-\frac{1}{2^{r-l-1}}$, the problem $\Pi^{r, l}(2, c)$ is NP-complete.

Proof: To prove the NP-completeness of $\Pi^{r, l}(2, c)$ we use a reduction from $\Pi^{r}(2)$, the problem of 2coloring unrestricted $r$-graphs. The latter problem is known to be NP-complete [20], but we include a simple proof here for self-containment.

Fact 3.2 For every $r \geq 3$ and $1 \leq l<r, \Pi^{r}(2)$ is $N P$-complete.

Proof: It is easy to see that $\Pi^{r}(2)$ is in NP. For the hardness part, we use a reduction from the so-called Not-All-Equal-rSAT (NAE- $r$ SAT) problem, in which, for a given boolean formula $\phi$ that is an instance of $r$-SAT we ask whether there is a truth-value assignment for the variables of $\phi$ such that each of its clauses has at least one true literal and at least one false literal. This problem is NP-complete [11]. For $2 \leq h \leq r$, let an $(h, r)$-graph be a hypergraph, whose hyperedges are of size $h$ or $r$ only. We denote the problem of 2-coloring $(h, r)$-graphs by $\Pi^{(h, r)}(2)$. Below we first show how NAE- $r$ SAT reduces in polynomial time to the problem $\Pi^{(2, r)}(2)$. Altogether, the proof will then be deduced from the following chain of reductions.

$$
\text { NAE- } r \text { SAT } \leq_{P} \Pi^{(2, r)}(2) \leq_{P} \Pi^{(3, r)}(2) \leq_{P} \cdots \leq_{P} \Pi^{(r, r)}(2)=\Pi^{r}(2)
$$

Given a boolean formula $\phi$, which is an instance of NAE- $r$ SAT construct a hypergraph $H^{\prime}=\left(V^{\prime}, E^{\prime}\right)$ which is a $(2, r)$-graph as follows. The set $V^{\prime}$ contains a vertex, both, for each variable and each complement, whereas the edge set $E^{\prime}$ consists of $r$-tuples corresponding to all clauses of $\phi$ and all pairs of the form $\{x, \bar{x}\}$. Clearly, $\phi$ is satisfiable by an assignment in which there is at least one true literal and at least one false literal in every clause whenever $H^{\prime}$ is 2-colorable with color classes corresponding to the truth values of literals.

For the remaining reductions we just show the first step $\Pi^{(2, r)}(2) \leq_{P} \Pi^{(3, r)}(2)$. Let $H^{\prime}=\left(V^{\prime}, E^{\prime}\right)$ be a $(2, r)$-graph and $H^{\prime \prime}=(V, F)$ be a $(3, r)$-graph in which we keep the vertices of $H^{\prime}$ and all its edges of size $r$. In addition, every pair $\{u, v\}$ which is an edge of $H^{\prime}$ is replaced by a gadget consisting of three additional vertices $\{r, s, t\}$ and four edges formed by the following triples $\{r, s, t\},\{u, v, r\}$, $\{u, v, s\},\{u, v, t\}$. Obviously, $H^{\prime}$ is 2-colorable if and only if $H^{\prime \prime}$ is 2-colorable. In the same way, one can gradually reduce the starting problem to $\Pi^{r}(2)$ replacing the edges of size $i$ by $i+2$ edges of size $i+1$ for $2 \leq i \leq r-1$.

Returning to the proof of Lemma 3.1, we next show a reduction from $\Pi^{r}(2)$ to $\Pi^{r, l}(2, c)$. Let $H=$ $(V, E)$ be an $r$-graph on $|V|=n$ vertices. Fix $\epsilon>0$ and let $N=\left\lceil\frac{n}{\epsilon}\right\rceil$. We construct an $r$-graph $H^{\prime}=\left(V^{\prime}, E^{\prime}\right)$ with $V^{\prime}=V_{0} \cup V_{1} \cup V_{2}$, where $V_{0}=V$ and $\left|V_{i}\right|=N$ for $i=1,2$. The edge set is $E^{\prime}=E \cup E_{012} \cup E_{12}$, where $E_{012}$ corresponds to all $r$-tuples of vertices in $V^{\prime}$ that intersect all sets $V_{0}, V_{1}$ and $V_{2}$, whereas $E_{12}$ contains all $r$-tuples of vertices in $V^{\prime}$ intersecting $V_{1}$ and $V_{2}$ but not $V_{0}$ (see 


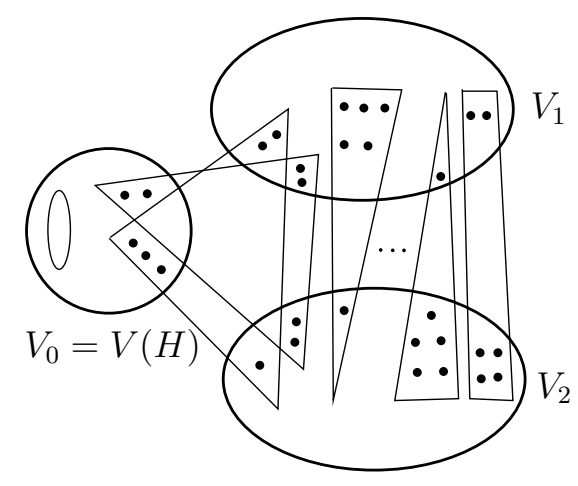

Fig. 1: The gadget $(r=6)$.

Fig 1). Notice that $\left|V^{\prime}\right|=2 N+n$ and

$$
\begin{aligned}
\delta_{l}\left(H^{\prime}\right) & =\sum_{i=1}^{r-l}\left(\begin{array}{c}
N \\
i
\end{array}\right)\left(\begin{array}{c}
N \\
r-l-i
\end{array}\right)=\left(\begin{array}{c}
2 N \\
r-l
\end{array}\right)-2\left(\begin{array}{c}
N \\
r-l
\end{array}\right) \\
& \geq\left(1-\frac{1}{2^{r-l-1}}-\epsilon\right)\left(\begin{array}{c}
2 N+n \\
r-l
\end{array}\right)
\end{aligned}
$$

because $N \geq \frac{n}{\epsilon}$.

Now, if $H$ admits a 2-coloring, say in red and blue, then the coloring of $H$ can be extended to $H^{\prime}$ by assigning blue to all vertices in $V_{1}$ and red to all vertices in $V_{2}$. On the other hand, if $H^{\prime}$ admits a 2-coloring, then, trivially, $H$ has a 2-coloring.

\section{2-coloring Algorithm}

In this section we present a polynomial time algorithm for the problem $\Pi^{r, l}(2, c)$, with $c>1-\frac{1}{2^{r-l-1}}$, thereby completing the proof of Theorem 1.3 First we outline the general idea of the algorithm and introduce the necessary terminology.

\subsection{General Framework}

In his paper [8], Edwards proposes a polynomial time algorithm for $k$-coloring any graph $G=(V, E)$ with the minimum degree at least $c|V(G)|$ for $c>\frac{k-3}{k-2}$ and $k \geq 3$. The main idea of this algorithm relies on the fact that with the prescribed minimum degree bound every vertex of $V$ contains in its neighborhood many $(k-2)$-cliques. Therefore, it is possible to find a $O(\log n)$-sized set $K \subset V(G)$ with the property that the neighborhood of every vertex, induced on $K$, contains at least one $K_{k-2}$. One can then search all $k$-colorings of the induced subgraph $G[K]$, whose number is $k^{O(\log n)}$, in polynomial time. Next, Edwards uses the fact that, by the construction, every admissible and effectively constructible $k$-coloring of $G[K]$ leaves at most two colors available for every vertex outside $K$. This, in turn, allows him to use 
2-SAT to verify in polynomial time whether or not a $k$-coloring of $G[K]$ can be extended to a $k$-coloring of the whole graph $G$. It is well known that 2-SAT is in P (see [10]) and the best algorithm is linear.

In the generalization of this idea to $r$-graphs, instead of considering subgraphs induced by neighborhoods of vertices, we propose to use the notion of a link in a hypergraph. Suppose $H$ is an $r$-graph and $v \in V(H)$. The link (neighborhood) graph of $v$ is the $(r-1)$-graph $G(v)=\left\{\left\{x_{1}, \ldots, x_{r-1}\right\}\right.$ : $\left.\left\{x_{1}, \ldots, x_{r-1}, v\right\} \in H\right\}$. If $r=3$ then the link graph of every vertex in $V(H)$ is a graph. This can be generalized to a set $S \subseteq V$ of $l$ vertices. For $0<l \leq r-1$, the link graph of $S \in\left(\begin{array}{c}V(H) \\ l\end{array}\right)$ is an $(r-l)$-graph $G(S)=\left\{\left\{x_{1}, \ldots, x_{r-l}\right\}:\left\{x_{1}, \ldots, x_{r-l}\right\} \cup S \in H\right\}$.

Also, instead of using cliques in the neighborhoods of vertices, we look for members of a family $\mathcal{F}$ of non-bipartite $(r-l)$-graphs in link graphs. In brief, our algorithm constructs in $H$ a $O(\log n)$-sized subset $K \subset V(H)$ such that the link of every vertex or pair of vertices induced on $K$ contains at least one copy of an $(r-l)$-graph from $\mathcal{F}$. Then, for every admissible 2-coloring of $H[K]$, it tests whether or not this coloring can be extended to the rest of $H$.

The coloring algorithms given in this paper share the following general framework.

ALGORITHM COLOR(outline)

In: an $r$-graph $H=(V, E)$ with $|V(H)|=n$ and $\delta_{l}(H) \geq c\left(\begin{array}{c}n-l \\ r-l\end{array}\right)$, and an integer $k$

Out: YES, if $H$ has a (strong) $k$-coloring; NO, otherwise

1. Choose a suitable family $\mathcal{F}$ of non-bipartite $(r-l)$-graphs.

2. Find a $O(\log n)$-sized subset $K \subset V(H)$ such that the link of every vertex $(l=1)$ or pair of vertices $(l=2)$ of $H$ induced by $K$ contains at least one copy of $F \in \mathcal{F}$.

3. For every $k$-coloring $f$ of $H[K]$ do

(a) Check if $f$ can be extended to $V(H)-K$ using properties of $\mathcal{F}$ and possibly 2-SAT.

(b) If successful then return YES (and present the final (strong) $k$-coloring).

4. If no $k$-coloring found, return NO.

Observe that the core $K$ is small enough to efficiently search through all possible assignments of colors to its vertices. What is more, any admissible coloring of the core reduces either the number of colors available to a vertex $(l=1)$, or the number of combinations of 2-colorings of every pair of vertices $(l=2$ ) outside $K$. In the former case, Step 3(a) boils down to a mere checking if the unique extension of $f$ is admissible. In the latter case a reduction to 2-SAT is used to find a final coloring. As we shall see soon, it is therefore desirable that the family $\mathcal{F}$ consists of $(r-l)$-graphs that are non-bipartite. Besides, to be able to find a small core we require that there are sufficiently many copies of graphs in $\mathcal{F}$ contained in the links of all vertices or pairs of vertices. The construction of the core relies on the following property of bipartite graphs.

Lemma 4.1 For every bipartite graph $B=(X \cup Y, E)$ such that $d_{B}(v) \geq \beta|Y|$, for all $v \in X$ there exists a set of vertices $D \subseteq Y$ of size at most $\log _{1 /(1-\beta)}|X|+1$ that dominates $X$. Moreover, $D$ can be constructed in $O\left(\log _{1 /(1-\beta)}|X|\right)$ rounds, each of them having a number of steps which is polynomial in $|X| \cdot|Y|$. 
Proof: We construct $D$ by sequentially adding to it vertices of large degree. Since the degree of every vertex $v$ in $X$ is at least $\beta|Y|$, there exists a vertex $w \in Y$ such that $d_{B}(w) \geq \beta|X|$ and we add it to $D$. Observe that $w$ covers at least $\beta|X|$ vertices of $X$, which we remove from $X$ while $w$ is removed from $Y$. In the remaining graph, every vertex in $X$ still has a degree of at least $\beta|Y|$ and the same argument can be repeated until all vertices in $X$ are covered, which happens after $O\left(\log _{1 /(1-\beta)}|X|\right)$ rounds. As a result, every vertex in $X$ has a neighbor in $D$.

After appropriately defining the auxiliary bipartite graph, the set $D$ gives rise to the core $K$. In order to guarantee its existence the degree condition must be satisfied. This, in turn, will follow from a bound on $\delta_{l}(H)$ and some properties of dense hypergraphs.

\subsection{Dense Hypergraphs are not Bipartite}

We start by showing that $r$-graphs with sufficiently many edges are not bipartite. For an $r$-graph $H$ on $m$ vertices, let $\varrho(H)=\frac{e(H)}{\left(\begin{array}{c}m \\ r\end{array}\right)}$ denote the density of $H$.

Observation 4.2 For $s \geq 3, \epsilon>0$ and $m=\left\lceil\frac{4}{\epsilon}\right\rceil>2 s$ no s-graph on $m$ vertices with density at least $1-\frac{1}{2^{s-1}}+\frac{\epsilon}{2}$ is bipartite.

Proof: Fix $s \geq 3, \epsilon>0$ and $m=\left\lceil\frac{4}{\epsilon}\right\rceil$. Without loss of generality assume that $m$ is even and observe that every bipartite $s$-graph with bipartition $V=A \cup B,|A|=a$ and $|B|=m-a$ has at most $\left(\begin{array}{c}m \\ s\end{array}\right)-2\left(\begin{array}{c}m / 2 \\ s\end{array}\right)$ edges. Indeed, $e(G)=\sum_{i=1}^{s-1}\left(\begin{array}{c}a \\ i\end{array}\right)\left(\begin{array}{c}m-a \\ s-i\end{array}\right)=\left(\begin{array}{c}m \\ s\end{array}\right)-\left(\begin{array}{c}a \\ s\end{array}\right)-\left(\begin{array}{c}m-a \\ s\end{array}\right)$ is maximized for $a=\frac{m}{2}$. Hence,

$$
\begin{aligned}
\varrho(G) & \leq 1-2 \frac{\left(\begin{array}{c}
m / 2 \\
s
\end{array}\right)}{\left(\begin{array}{c}
m \\
s
\end{array}\right)} \leq 1-2^{1-s}\left(1-\frac{(s-1)^{2}}{m-s+1}\right) \\
& \leq 1-\frac{1}{2^{s-1}}+\frac{2(s-1)^{2}}{m 2^{s-1}} \leq 1-\frac{1}{2^{s-1}}+\frac{2}{m} .
\end{aligned}
$$

Another useful observation states that a dense $s$-graph has many induced $s$-subgraphs of any given size, which are almost as dense as itself.

Observation 4.3 For $q, s \geq 3$ and $\rho, \epsilon>0$, every s-graph $H$ on $n \geq q$ vertices with density $\varrho(H) \geq$ $\rho+\epsilon$ contains at least $\frac{\epsilon}{2}\left(\begin{array}{l}n \\ q\end{array}\right)$ induced q-vertex subgraphs $H^{\prime}$ with density $\varrho\left(H^{\prime}\right) \geq \rho+\frac{\epsilon}{2}$.

Proof: Let $s \geq 3$ and $H$ be an $s$-graph on $n \geq q$ vertices with density at least $\rho+\epsilon>0$. While considering induced $s$-subgraphs $H^{\prime}$ of $H$ on $q$ vertices, we have

$$
\sum_{\substack{H^{\prime} \subset \text { ind } \\
\left|H^{\prime}\right|=q}} e\left(H^{\prime}\right)=e(H)\left(\begin{array}{c}
n-s \\
q-s
\end{array}\right) \geq(\rho+\epsilon)\left(\begin{array}{c}
n \\
s
\end{array}\right)\left(\begin{array}{l}
n-s \\
q-s
\end{array}\right) .
$$

The above inequality implies that there are at least $\frac{\epsilon}{2}\left(\begin{array}{l}n \\ q\end{array}\right)$ induced subgraphs $H^{\prime} \subset_{\text {ind }} H$ on $q$ vertices with density at least $\rho+\frac{\epsilon}{2}$. Indeed, suppose this is not true. Then

$$
\sum_{\substack{H^{\prime} \subset \text { ind } H \\
\left|H^{\prime}\right|=q}} e\left(H^{\prime}\right)<\frac{\epsilon}{2}\left(\begin{array}{l}
n \\
q
\end{array}\right)\left(\begin{array}{l}
q \\
s
\end{array}\right)+\left(\rho+\frac{\epsilon}{2}\right)\left(\begin{array}{l}
n \\
q
\end{array}\right)\left(\begin{array}{l}
q \\
s
\end{array}\right)=(\rho+\epsilon)\left(\begin{array}{l}
n \\
s
\end{array}\right)\left(\begin{array}{c}
n-s \\
q-s
\end{array}\right),
$$


which is a contradiction.

\subsection{The Algorithm}

Now we show how to put together the ideas introduced above into a polynomial time algorithm for 2coloring $r$-graphs, in which vertex or pair degrees are large. Our description will specify how the particular steps of the general framework introduced in the previous section should be implemented. First, we recall the theorem given in [4], which is a special case of the second part of our Theorem 1.3, and give an alternative proof of it (Theorem $1.5 \mathrm{a}$ )). As a result we get a 2-coloring algorithm for $\Pi^{3,2}(2, c)$, which, in contrast to the algorithm given in [4], is deterministic and has slightly better running time.

Theorem 4.4 (Th.3, [4]) For every $c>0$, the problem $\Pi^{3,2}(2, c)$ has time complexity

$O\left(\max \left\{n^{3} \log n ; n^{\frac{2}{\log (1-c)^{-1}}+1}\right\}\right)$.

Proof: Let $H$ be a 3-graph on $n$ vertices with $\delta_{2}(H) \geq c n$ and $c>0$. The proof follows the general framework given in Section 4.1 .

Step 1. Note that the link graph of every pair of vertices is just a set of vertices. Therefore, the choice of the family $\mathcal{F}$ trivializes to a single vertex.

Step 2. The selection of the core, which was randomized in [4], can be made deterministic using Lemma 4.1 For this, we construct a bipartite graph $B=(X \cup Y, E)$ with the set $X$ corresponding to all pairs of vertices of $H$, and $Y$ to all vertices of $H$. We put an edge between $x=\{u, v\} \in X$ and $w \in Y$ in $E(B)$ if $\{u, v, w\} \in H$. By the assumption $\delta_{2}(H) \geq c(n-1)$, it holds that $\delta_{B}(x) \geq c(n-1)$ for every $x \in X$ and therefore, via Lemma 4.1 we can find in $O\left(n^{3} \log _{(1-c)^{-1}} n\right)$ steps a set of vertices $D \subset V(H)$ of size at $\operatorname{most} \log _{(1-c)^{-1}} n+1$ such that every pair of vertices in $H$ has a neighbor in $D$. Let $K:=D$.

Step 3. Consider a proper 2-coloring $f$ of the hypergraph $H[K]$. Denote by $A \cup B$ the color classes of $f$ and let $K_{A}=K \cap A$ and $K_{B}=K \cap B$. Since every pair of vertices $\{u, v\}$ outside $K$ has a neighbor $w \in K$, the vertices $u$ and $v$ cannot both have the color $f(w)$. This observation is used to extend the 2-coloring of the core to $V(H)-K$ using an instance of 2-SAT, $\phi(f)$. Let $V(H)-K=\left\{v_{1}, \ldots, v_{m}\right\}$ and assign a variable $x_{i}$ to represent the fact that , $f\left(v_{i}\right) \in A$ ” for every $1 \leq i \leq m$. The formula $\phi(f)$ contains the following clauses.

- $\bar{x}_{i} \vee \bar{x}_{j}$, for $1 \leq i<j \leq m$ if $N\left(v_{i}, v_{j}\right) \cap K_{A} \neq \emptyset, N\left(v_{i}, v_{j}\right) \cap K_{B}=\emptyset$, and

- $x_{i} \vee x_{j}$, for $1 \leq i<j \leq m$ if $N\left(v_{i}, v_{j}\right) \cap K_{A}=\emptyset, N\left(v_{i}, v_{j}\right) \cap K_{B} \neq \emptyset$.

Notice that even if $\phi(f)$ is satisfiable, it does not yet guarantee that there are no monochromatic edges outside $K$. However, if $f$ coincides on $K$ with $\chi$, a proper 2-coloring of $H$, then the solution to $\phi(f)$ always yields a coloring which differs on at most one vertex in each color class from $\chi$. To see this, suppose that $\phi(f)$ imposes a coloring, in which two vertices $v_{1}, v_{2} \in V(H)-K$ are such that $f\left(v_{1}\right)=$ $f\left(v_{2}\right) \in A$, whereas $\chi\left(v_{1}\right)=\chi\left(v_{2}\right) \in B$. But then, for every $w \in N\left(v_{1}, v_{2}\right) \cap K$, we have $\chi(w) \in$ $K_{A}$ and thus $f(w) \in K_{A}$. Hence, the triple $\left(v_{1}, v_{2}, w\right)$ is monochromatic, which is impossible by the definition of $\phi(f)$. The coloring yielding monochromatic edges can be corrected easily by considering all pairs of vertices in $V(H)-K$ of opposite colors and changing one of their colors, or both, if necessary. In the case in which $\phi(f)$ is not satisfiable, another 2-coloring $f$ has to be considered. If no such coloring 
exists then $H$ is not 2-colorable. Since there are at most $2^{\log _{(1-c)^{-1}} n^{2}}=n^{\frac{2}{\log (1-c)^{-1}}}$ possible 2-colorings of $H[K]$, the running time of our algorithm is $O\left(\max \left\{n^{3} \log _{(1-c)^{-1}} n ; n^{\frac{2}{\log (1-c)^{-1}}+1}\right\}\right)$, which for $c<\frac{1}{2}$ is dominated by $O\left(n^{\frac{2}{\log (1-c)^{-1}}+1}\right)$.

Now we present the algorithm in a general setting.

Lemma 4.5 For every $r \geq 3$ and $l=1,2, \Pi^{r, l}(2, c)$ has time complexity $O\left(\max \left\{n^{1 / \epsilon+l} \log n ; n^{\frac{4 l}{\epsilon \log (1-\epsilon / 2)^{-1}}+1}\right\}\right)$, where $\epsilon=c-1+\frac{1}{2^{r-l-1}}$.

\section{Proof:}

Fix $r \geq 3, l=1,2$ and $\epsilon>0$, and let $H$ be an instance of $\Pi^{r, l}(2, c)$, i.e. an $r$-graph with $|V(H)|=$ $n, \delta_{l}(H) \geq c\left(\begin{array}{c}n-l \\ r-l\end{array}\right)$ and $c>1-\frac{1}{2^{r-l-1}}$.

Step 1. Let $\mathcal{F}$ be the family of $(r-l)$-graphs $F$ on $m=\lfloor 4 / \epsilon\rfloor$ vertices with density $\varrho(F) \geq 1-$ $\frac{1}{2^{r-l-1}}+\frac{\epsilon}{2}$, which are non-bipartite by Observation 4.2 with $s=r-l$.

Step 2. Construct a core in $H$ using Lemma 4.1 Notice that, for every vertex or pair of vertices in $V$, their corresponding link graph is an $(r-l)$-graph on $n-l$ vertices and by the assumption on $\delta_{l}(H)$, contains at least $\left(1-\frac{1}{2^{r-l-1}}+\epsilon\right)\left(\begin{array}{l}n-l \\ r-l\end{array}\right)$ edges. Consequently, by Observation 4.3 with $s=r-l$ and $q=m$, each link graph contains at least $\frac{\epsilon}{2}\left(\begin{array}{c}n-l \\ m\end{array}\right)$ induced subgraphs $F \in \mathcal{F}$ which are $(r-l)$-graphs on $m$ vertices with density at least $\rho_{r-l}+\frac{\epsilon}{2}$.

We build an auxiliary bipartite graph $B=(X \cup Y, E)$ with $X=\left(\begin{array}{c}V(H) \\ l\end{array}\right)$ and $|Y|=\left(\begin{array}{c}V(H) \\ m\end{array}\right)$. We put an edge $e=S U$ in $E(B)$ for $S \in\left(\begin{array}{c}V(H) \\ l\end{array}\right)$ and $U \in Y$ whenever $\rho(G(S)[U]) \geq \rho_{r-l}+\frac{\epsilon}{2}$. It follows that $\delta_{B}(S) \geq \beta|Y|$ with $\beta=\frac{\epsilon}{2}$. We can therefore construct in time $O\left(n^{m+l} \log _{1 /\left(1-\frac{\epsilon}{2}\right)} n^{l}\right)$ a subset $D$ of $Y$ of size at $\operatorname{most}^{\log _{1 /\left(1-\frac{\epsilon}{2}\right)}} n^{l}+1$ that dominates every element in $X$. Then $K=\bigcup_{U \in D} U$ is the core with the property that the link of every set of $l$ vertices in $V(H)-K$ induced on $K$ will contain at least one copy of a non-bipartite $(r-l)$-graph $F \in \mathcal{F}$. Note that $|K| \leq m l \log _{1 /\left(1-\frac{\epsilon}{2}\right)} n$.

Step 3. By the construction in Step 2, every 2-coloring of $H[K]$ yields a monochromatic edge in every link and it is possible to check if it can be extended to the vertices outside the core. If $l=1$ then any admissible 2-coloring of the vertices of the core leaves at most one color available for every vertex $v \in V(H)-K$. If $l=2$ then we can search for the extension of any 2-coloring of the core using 2-SAT in the same way as in the proof of Theorem 4.4 Moreover, since $|K| \leq m l \log _{(1-\epsilon / 2)^{-1}} n$, there are at most $n^{\frac{4 l}{\epsilon \log (1-\epsilon / 2)^{-1}}}$ colorings of the core and this step has running time $O\left(n^{\frac{4 l}{\epsilon \log (1-\epsilon / 2)^{-1}}+1}\right)$.

Summarizing, Lemma 4.5 and Lemma 3.1 yield Theorem 1.3 . From the proof of Lemma 4.5 one can see that the closer the minimum degree of the input is to the threshold value, the more costly it is to decide whether it is 2-colorable. Indeed, if $\epsilon \rightarrow 0$, then the running time of the algorithm for $\Pi^{r, l}(2, c)$ becomes $O\left(n^{8 / \epsilon^{2}+1}\right)$. This, however, can be improved in some cases as indicated in Theorem 1.5 . The proof of this statement relies heavily on some nontrivial results on Turán numbers for graphs and hypergraphs. Before we show the proof, we recall in the next section some important facts about Turán numbers. 


\subsection{Turán Numbers for Graphs and Hypergraphs}

Given an $r$-graph $F$, let ex $(n, F)$, the Turán number of $F$, be the maximum number of edges in an $n$ vertex $r$-graph with no copy of $F$. The limit $d_{r}(F)=\lim _{n \rightarrow \infty} \frac{e x(n, F)}{\left(\begin{array}{l}n \\ r\end{array}\right)}$ is referred to as the Turán density. In the case in which $F$ is a complete graph on $q$ vertices, $F=K_{q}$, we denote the Turán density by $d_{r}(q)$. In 1941 Turán proved that for $q \geq 3$ it holds $d_{2}(q)=\frac{q-2}{q-1}$ (see [6] for a new proof).

Not much is known about Turán numbers for hypergraphs. One special case which we use here is the 3-graph called Fano plane. The Fano plane $F$ is the projective plane over the field with two elements. It has seven vertices, which can be identified with the non-zero vectors of length three. It has seven edges, corresponding to the lines of the plane. A triple $x y z$ is an edge if $x+y=z$. The Fano plane requires three colors for a proper coloring and therefore no 2-colorable hypergraph can contain it as a subgraph.

By the result of De Caen and Füredi [5] it is known that the Turán density of the Fano plane is $3 / 4$. Subsequently, Keevash and Sudakov in [18] determined the exact Turán number of the Fano plane $F$ as

$$
e x(n, F)=\left(\begin{array}{c}
n \\
3
\end{array}\right)-\left(\begin{array}{c}
\left\lfloor\frac{n}{2}\right\rfloor \\
3
\end{array}\right)-\left(\begin{array}{c}
\left\lceil\frac{n}{2}\right\rceil \\
3
\end{array}\right) .
$$

Furthermore, there is a useful fact, known also as a supersaturation result, saying that $r$-graphs which exceed Turán density for a given $r$-graph contain already many copies of the $r$-graph. It was proved by Bollobás in [3, p.307] for graphs and by Erdös and Simonovits [9], for $r$-graphs. We use a version of this result by Keevash [17], which allows to estimate the implicit constant whenever the corresponding Turán number is known.

Lemma 4.6 ([17]) Let $H$ be an r-graph on $n \geq n_{0}$ vertices and let $F$ be an $r$-graph on $v_{F}$ vertices. For every $\epsilon>0$ and an integer $t$ the following holds. If $e(H) \geq\left(d_{r}(F)+\epsilon\right)\left(\begin{array}{l}n \\ r\end{array}\right)$ and ex $(t, F) \leq$ $\left(d_{r}(F)+\frac{\epsilon}{2}\right)\left(\begin{array}{l}t \\ r\end{array}\right)$, then $H$ contains at least $\frac{\epsilon}{2\left(\begin{array}{c}t \\ v_{F}\end{array}\right)}\left(\begin{array}{c}n \\ v_{F}\end{array}\right)$ copies of $F$.

The above lemma together with formula (1) has the following implication for the Fano plane that we use later.

Corollary 4.7 For $r=3$ and the Fano plane F, it holds

$$
e x(t, F) \leq\left(\frac{3}{4}+\frac{\epsilon}{2}\right)\left(\begin{array}{l}
t \\
3
\end{array}\right), \text { for } t \geq \frac{1}{\epsilon} .
$$

Thus, every 3-graph $H$ with $e(H) \geq\left(\frac{3}{4}+\epsilon\right)\left(\begin{array}{c}n \\ 3\end{array}\right)$ contains at least $7 ! \cdot \frac{\epsilon^{8}}{2}\left(\begin{array}{l}n \\ 7\end{array}\right)$ copies of F.

Lemma 4.6 applies also to triangles $\left(F=K_{3}\right)$ but we use here a result of Goodman [12] (see also Razborov [24]), which gives a much better bound.

Fact 4.8 ([12]) For every $\epsilon>0$, a graph $G$ on $n$ vertices and with $e(G) \geq\left(\frac{1}{2}+\epsilon\right)\left(\begin{array}{l}n \\ 2\end{array}\right)$ contains at least

$$
\left(\frac{1}{2}+\epsilon\right) 2 \epsilon\left(\begin{array}{l}
n \\
3
\end{array}\right) \geq \epsilon\left(\begin{array}{l}
n \\
3
\end{array}\right)
$$

triangles. 


\subsection{The Proof of Theorem 1.5 b) and c)}

In the proof of this theorem we apply a similar concept as in the proof of Lemma 4.5. In particular, the general framework of the algorithm is the same but the constructions of the core is more efficient. For the ease of presentation we concentrate on the case $c$ ) in which $l=1$ and $r=4$, i.e. we show that for every $c>\frac{3}{4}$, the problem $\Pi^{4,1}(2, c)$ has time complexity $O\left(\max \left\{n^{8} \log n ; n^{\frac{7}{\log \left(1-\epsilon^{8} / 2\right)^{-1}}+1}\right\}\right)$, where $\epsilon=c-\frac{3}{4}>0$.

Proof: Let $H$ be a 4-graph with $\delta_{1}(H)>\frac{3}{4}\left(\begin{array}{c}n-1 \\ 3\end{array}\right)$.

Step 1. Let $\mathcal{F}$ contain just the Fano plane.

Step 2. Construct a core in $H$ using an auxiliary bipartite graph $B=(X \cup Y, E)$. Take $X=$ $V(H), Y=\left(\begin{array}{c}V(H) \\ 7\end{array}\right)$ and put an edge $e=v U$ in $E(B)$ for $v \in V(H)$ and $U \in Y$ whenever $G(v)[U]$ contains the Fano plane. To construct a core using Lemma 4.1 we need to make a few observations first. By assumption $\delta_{1}(H) \geq\left(\frac{3}{4}+\epsilon\right)\left(\begin{array}{c}n-1 \\ 3\end{array}\right)$ for some $\epsilon>0$, and, for every $v \in V$, its link graph $G(v)$ is a 3-graph such that $|E(G(v))| \geq\left(\frac{3}{4}+\epsilon\right)\left(\begin{array}{c}n-1 \\ 3\end{array}\right)$. Using Corollary 4.7 , for every $v \in V(H)$ we are guaranteed at least $7 !\left(\epsilon^{8} / 2\right)\left(\begin{array}{l}n \\ 7\end{array}\right)$ copies of the Fano plane in its link graph $G(v)$ and at most $7 !$ of them can share the same vertex set. Hence, applying Lemma 4.1 to $B$ with $\beta=\epsilon^{8} / 2$ we are able to find a $\log _{1 /\left(1-\epsilon^{8} / 2\right)} n$-sized subset $D$ of $Y$ that dominates every vertex in $V(H)$. Thus, for every vertex $v \in V(H)$ there is at least one copy of the Fano plane in its link induced by the core. This step has time complexity $O\left(n^{8} \log _{1 /\left(1-\epsilon^{8} / 2\right)} n\right)$.

Step 3. We now proceed by sequentially considering 2-colorings of the hypergraph induced by the vertices of the core $K:=\bigcup_{U \in D} U$. Notice that $|K| \leq 7 \log _{1 /\left(1-\epsilon^{8} / 2\right)} n$ and the number of 2-colorings of $H[K]$ is at most $n^{\frac{7}{\log \left(1-\epsilon^{8} / 2\right)^{-1}}}$.

If no proper 2-coloring of $H[K]$ can be found then $H$ is not 2-colorable. Otherwise, if a proper 2coloring of $H[K]$ is encountered we check if it can be extended to the vertices outside $K$. Notice that for every 2-coloring of the Fano plane there is at least one monochromatic edge. In consequence, by our construction, every vertex in $V(H)-K$ has at most one color available. This implies that it can be verified in polynomial time if every proper coloring of the core with two colors can be extended to the remaining vertices and $\Pi^{4,1}(2, c)$ is in P. Accordingly, its time complexity is determined by the construction of the core out of the Fano plane and the number of possible 2-colorings of the core. Thus, it is $O\left(\max \left\{n^{8} \log n ; n^{\frac{7}{\log \left(1-\epsilon^{8} / 2\right)^{-1}}+1}\right\}\right)$. As a matter of fact, this running time depends on the constant in Corollary 4.7 On that ground, any improvement of that constant $\left(\epsilon^{8} / 2\right)$ would forthwith lead to an improvement of time complexity of $\Pi^{4,1}(2, c)$ (and $\Pi^{5,2}(2, c)$ ).

The same reasoning yields the proof for $\Pi^{3,1}(2, c)$ with $c>\frac{1}{2}$. In this case we set $\mathcal{F}=\left\{K_{3}\right\}$. By Fact 4.8 for every $v \in V$, its link graph $G(v)$ contains enough edges to guarantee $\epsilon\left(\begin{array}{l}n \\ 3\end{array}\right)$ triangles for $\epsilon=c-\frac{1}{2}$. Thus, using Lemma 4.1 we can find a core $K$ of at most $3 \log _{(1-\epsilon)^{-1}} n$ vertices such that for any $v \in V(H)$ the link $G(v)[K] \supset K_{3}$. Hence, there are at most $n^{\frac{3}{\log (1-\epsilon)^{-1}}}$ possible 2-colorings of $H[K]$. The rest of the proof follows in the similar way as before and overall, the running time of the algorithm in this case achieves $O\left(\max \left\{n^{3} \frac{\log n}{\log (1-\epsilon)^{-1}} ; n^{\frac{3}{\log (1-\epsilon)^{-1}}+1}\right\}\right)$, which is better than that in Lemma 4.5 . 
Combining the ideas from the above proofs which rely on Turán numbers for the triangle and the Fano plane with the 2-SAT formulation suggested by Chen and Frieze (see the proof of Theorem 4.4), it is straightforward to deduce the polynomial algorithms for $\Pi^{4,2}(2, c)$ when $c>\frac{1}{2}$ and $\Pi^{5,2}(2, c)$ when $c>$ $\frac{3}{4}$.

Observe that the main difference between the above proof and the proof of Theorem 1.3 is that here we know explicitly the non-bipartite $(r-l)$-graph of the desired Turán density $1-\frac{1}{2^{r-l-1}}$, which has a small number of vertices (seven for the Fano plane). In the general result we are only guaranteed the existence of non-bipartite $(r-l)$-graphs on $m$ vertices but we have to search for them and $m$ can be a large constant growing with $1 / \epsilon$. Nevertheless, had, for instance, a non-bipartite 4 -graph of Turán density $7 / 8$ been found, a better running time of the algorithm for $\Pi^{5,1}(2, c)$ and $\Pi^{6,2}(2, c)$ could be established.

\subsection{Enumeration problems}

The ideas presented so far in this section for polynomial 2-coloring algorithms can be also used for enumerating the colorings of hypergraphs with the same degree bounds. Formally, we can define the counting problem in the following way.

Definition 4.9 For fixed integers $r, 1 \leq l \leq r-1$ and $k$, and a real number $0 \leq c \leq 1$, define the problem $\# \Pi^{r, l}(k, c)$ as follows:

Input: $r$-graph $H=(V, E)$ with $|V(H)|=n$ and $\delta_{l}(H) \geq c\left(\begin{array}{c}n-l \\ r-l\end{array}\right)$

Output: the number of $k$-colorings of $H$.

In general this problem is harder than its decision version, but from Lemma 4.5 we can easily deduce the following result.

Corollary 4.10 For $r \geq 3$ the problem $\# \Pi^{r, 1}(2, c)$ is polynomial, for $c>1-\frac{1}{2^{r-2}}$.

Proof: Note that whenever $l=1$, we build a core of size $O(\log n)$ in the 2-coloring algorithm and the number of 2-colorings of $H[K]$ which have unique extension to $V(H)-K$ is at most $2^{O(\log n)}$. Thus, the counting problem is solvable in polynomial time.

\section{Strong Coloring}

In this section we restrict colorings to the strong ones. As mentioned in the Introduction (see Remark 1.7), a strong coloring of an $r$-graph can be seen as a proper coloring of the clique graph $G_{r}(H)$ obtained from $H=(V, E)$ by replacing each hyperedge with an $r$-clique. There is a relation stated below between the minimum vertex degree in a 3-graph $H$ and the minimum vertex degree in its clique graph $G_{3}(H)$.

Fact 5.1 For every $c>0$ and a 3-graph $H(V, E)$, if $\delta_{1}(H) \geq c\left(\begin{array}{c}n-1 \\ 2\end{array}\right)$ then $\delta\left(G_{3}(H)\right) \geq \sqrt{c}(n-1)$.

Proof: Let $v$ be a vertex in $V(H)$ of the minimum degree $\delta_{1}(H)$ and let $i_{v}$ be the number of isolated vertices in its link graph $G(v)$. Recall that the vertex set of $G(v)$ is $V(H)-\{v\}$. Observe that, because $(u, v) \in G_{3}(H)$ if and only if $u$ is not isolated in $G(v)$, we have $d_{G_{3}(H)}(v)=n-1-i_{v}:=x_{v}$. Thus, the smallest value of $x$ satisfying $c\left(\begin{array}{c}n-1 \\ 2\end{array}\right) \leq\left(\begin{array}{l}x \\ 2\end{array}\right)$ gives a lower bound on the minimum degree in $G_{3}(H)$. Since $\frac{x-1}{n-2} \leq \frac{x}{n-1}$, we have $x^{2} \geq c(n-1)^{2}$ and hence $\delta\left(G_{3}(H)\right) \geq x \geq \sqrt{c}(n-1)$.

The statement below is directly implied by Theorem 1.2 and Fact 5.1 
Corollary 5.2 For $k \geq 3$ and $c>\left(\frac{k-3}{k-2}\right)^{2}$, the problem $\Pi_{s}^{3}(k, c)$ is in $P$.

In the case of $k=4$, Corollary 5.2 guarantees a polynomial algorithm for $\Pi_{s}^{3}(4, c)$ when $c>\frac{1}{4}$. Theorem 1.9 stated in Introduction gives the same result for all values of $c>0$.

Proof of Theorem 1.9.: We follow the original idea of the proof by Edwards [8] and reduce the problem $\Pi_{s}^{3}(4, c)$ for $c>0$ to 2-SAT in polynomial time. The pseudocode of the algorithm is given below.

ALGORITHM STRONGCOLOR

In: a 3-graph $H$ with $\delta_{1}(H) \geq c\left(\begin{array}{c}n-1 \\ 2\end{array}\right)$, where $0<c \leq 1$.

Out: YES, if $H$ has a strong 4 -coloring; NO, otherwise

1. Let $\mathcal{F}$ consist of an edge $K_{2}$.

2. Build an auxiliary bipartite graph $B=(X \cup Y, E)$, where $X=V(H)$ and $Y=\left(\begin{array}{c}V(H) \\ 2\end{array}\right)$. For every $v \in X$ and edge $e \in Y$ we add an edge $v e \in E$ if $e \subseteq G(v)$. (Note that, for every $v \in X, d_{B}(v) \geq c|Y|$.) Using Lemma 4.1 find a subset $D \subseteq Y$ that dominates every vertex in $X$. Let $K=\bigcup_{e \in D} e$.

3. For every strong 4-coloring $f$ of $H[K]$ do

(a) Check if $f$ can be extended to $V(H)-K$, i.e. construct an instance $\phi(f)$ of 2-SAT on the set of vertices $V(H)-K$,

(b) If $\phi(f)$ is satisfiable then return YES (and present the final strong 4-coloring).

4. If no strong 4-coloring found, return NO.

Observe that Steps 2 and 3 can be performed in polynomial time.

The construction of $\phi(f)$ follows the idea of [8] and is different from the one applied to 2-colorings in the previous sections. For a fixed 4-coloring $f$ of $H[K]$ and every vertex $v \in V(H)-K$ let $S(v)$ be the set of admissible colors for $v$. Observe that as a consequence of Lemma 4.1 and $\delta_{1}(H) \geq c\left(\begin{array}{c}n-1 \\ 2\end{array}\right)$, there is at least one edge in the link of every vertex of $V(H)$ and thus two colors are already used, so $|S(v)| \leq 2$. Let $V(H)-K=\left\{v_{1}, \ldots, v_{m}\right\}$ and assign a variable $x_{i j}$ to represent the fact that "vertex $v_{i}$ has color $j$ " for every $1 \leq i \leq m, 1 \leq j \leq 4$. Now, $\phi(f)$ contains the following clauses

- $\left\{x_{i j} \mid j \in S\left(v_{i}\right)\right\}$, for $1 \leq i \leq m$ and

- $\bar{x}_{i j} \vee \bar{x}_{i h}$, for $1 \leq i \leq m, 1 \leq j<h \leq 4$ and

- $\bar{x}_{i j} \vee \bar{x}_{h j}, \bar{x}_{i j} \vee \bar{x}_{l j}, \bar{x}_{h j} \vee \bar{x}_{l j}$, for $\left\{v_{i}, v_{h}, v_{l}\right\} \in H$ and $1 \leq j \leq 4$.

It is worth noticing here that the above result does not necessarily imply that the problem of coloring clique graphs of hypergraphs with four colors is always polynomial.

On the hardness side, we have another result. 
Fact 5.3 For $k \geq 3$ and $c \leq \frac{(k-3)(k-4)}{(k-2)^{2}}$, the problem $\Pi_{s}^{3}(k, c)$ is NP-complete.

Proof: It is clear that $\Pi_{s}^{3}(k, c)$ is in NP. Now we show that it is NP-complete for $c=\frac{(k-3)(k-4)}{(k-2)^{2}}$. The proof uses a reduction from strong 3 -colorability, that is from the problem $\Pi_{s}^{3}(3)$. Strong 3 -coloring of a 3 -graph is NP-complete. This can be easily seen by considering a reduction from the NP-complete graph 3 -coloring problem, where every edge of a graph is replaced by a hyperedge with one new vertex added. Similarly, the problem $\Pi_{s}^{3}(4)$ is NP-complete. Hence, the cases of $k=3$ and $k=4$ (where $c=0$ ) follow.

To present the reduction $\Pi_{s}^{3}(3) \leq{ }_{P} \Pi_{s}^{3}(k, c)$, for $k \geq 5$, given a 3-graph $H=(V, E)$, where $|V(H)|=$ $n$ we construct a 3-graph $H^{\prime}=\left(V^{\prime}, E^{\prime}\right)$ in the following way. Into the set $V^{\prime}$ put $V_{0}=V$ and add sets $V_{1}, \ldots, V_{k-3}$ of new vertices such that $\left|V_{i}\right|=n$ for $i=1, \ldots, k-3$. Include $E$ into $E^{\prime}$ and add an edge for every triple of vertices $\{x, y, z\}$, such that $x \in V_{i}, y \in V_{j}, z \in V_{k}$, where $i, j, k$ are different indices from $\{0,1, \ldots, k-3\}$. Observe that $N=\left|V^{\prime}\right|=(k-2) n$ and the minimum degree

$$
\delta_{1}\left(H^{\prime}\right)=\frac{(k-3)(k-4) n^{2}}{2} \geq \frac{(k-3)(k-4)}{(k-2)^{2}}\left(\begin{array}{c}
N \\
2
\end{array}\right) .
$$

If $H$ is 3-colorable in the strong sense then, since $V_{1}, \ldots, V_{k-3}$ are independent sets, we can color each of them with a different color and hence $H^{\prime}$ admits a strong $k$-coloring this way. On the other hand, if $H^{\prime}$ can be colored using $k$ colors, in a strong way, then all vertices in the same set receive the same color and the colors used for the sets $V_{1}, \ldots, V_{k-3}$ must be all distinct. Therefore, only the same 3 colors are available for all vertices in $V_{0}$ and thus $H$ is strongly 3 -colorable.

Fact 5.3 and Corollary 5.2 together yield Proposition 1.8

\section{Final Remarks and Open Problems}

In this paper we have determined the complexity of the 2-coloring problem in $r$-graphs with prescribed $l$-wise degree, where $l=1,2$, strictly below and above the threshold value of $1-\frac{1}{2^{r-l-1}}$. Neither the hardness result nor the algorithmic part hold for $r$-graphs with minimum degree exactly on the threshold (except for $\Pi^{3,2}(2, c)$ ) and it would be interesting to see what is happening there.

While the hardness result holds for any $r \geq 3$ and $1 \leq l<r$, the idea of the polynomial algorithm breaks down for $l>2$ since the reduction to 2-SAT does not carry over. As we believe that Conjecture 1.4 is true, a new algorithmic idea is presumably needed to prove it.

Another wide open problem in this context is to extend Theorem 1.3 to more than two colors.

Regarding strong colorings, the complexity of $\Pi_{s}^{r}(k, c)$ is unknown for $r \geq 4$. Finally, the last open problem is to close the gap for $r=3$, namely, to determine the computational complexity of $\Pi_{s}^{3}(k, c)$ for $c \in\left[\frac{(k-3)(k-4)}{(k-2)^{2}},\left(\frac{k-3}{k-2}\right)^{2}\right]$ and $k \geq 5$.

\section{Acknowledgements}

I thank Marek Karpiński for bringing to my attention the result of Edwards and Peter Keevash for valuable suggestions. Also, I am grateful to the Editor for his numerous comments leading to the improvement of the exposition of the paper. 


\section{References}

[1] G. Agnarsson and M. M. Halldórsson. Strong colorings of hypergraphs. In G. Persiano and R. SolisOba, editors, WAOA 2004, volume 3351 of Lecture Notes in Computer Science, pages 253-266, 2005.

[2] F. Bernstein. Zur theorie der trigonometrische reihen. Leipz. Ber., 60:325-328, 1908.

[3] B. Bollobás. Extremal Graph Theory. Academic Press, 1978.

[4] H. Chen and A. Frieze. Coloring bipartite hypergraphs. In W. H. Cunningham, S.T. McCormick, and M. Queyranne, editors, Integer Programming and Combinatorial Optimization, volume 1084 of Lecture Notes in Computer Science, pages 345-358. Springer Berlin Heidelberg, 1996.

[5] D. De Caen and Z. Füredi. The maximum size of 3-uniform hypergraphs not containing a Fano plane. J. of Combinatorial Theory B, 78(2):274-276, 2000.

[6] R. Diestel. Graph Theory, 4th Edition, volume 173 of Graduate texts in mathematics. Springer, 2012.

[7] I. Dinur, O. Regev, and C. Smyth. The hardness of 3-uniform hypergraph coloring. Combinatorica, 25(5):519-535, 2005.

[8] K. Edwards. The complexity of colouring problems on dense graphs. Theoretical Computer Science, 43(C):337-343, 1986.

[9] P. Erdós and M. Simonovits. Supersaturated graphs and hypergraphs. Combinatorica, 3(2):181-192, 1983.

[10] S. Even, A. Itai, and A. Shamir. On the complexity of timetable and multicommodity flow problems. SIAM J.Comput., 5(4):691-703, 1976.

[11] M. R. Garey and D. S. Johnson. Computers and Intractability. Freeman, 1979.

[12] A. W. Goodman. On sets of acquaintances and strangers at any party. American Mathematical Monthly, 66(9):778-783, 1959.

[13] V. Guruswami, J. Hastad, and M. Sudan. Hardness of approximate hypergraph coloring. SIAM J. Comput., 31(6):1663-1686, 2002.

[14] M. Karpiński, A. Ruciński, and E. Szymańska. The complexity of perfect matching problems on dense hypergraphs. In Y. Dong, D. Du, and O. Ibarra, editors, Algorithms and Computation, volume 5878 of Lecture Notes in Computer Science, pages 626-636. Springer Berlin Heidelberg, 2009.

[15] M. Karpiński, A. Ruciński, and E. Szymańska. Computational complexity of the hamiltonian cycle problem in dense hypergraphs. In A. López-Ortiz, editor, LATIN 2010: Theoretical Informatics, volume 6034 of Lecture Notes in Computer Science, pages 662-673. Springer Berlin Heidelberg, 2010. 
[16] M. Karpiński, A. Ruciński, and E. Szymańska. Computational complexity of the perfect matching problem in hypergraphs with subcritical density. International J. of Foundations of Computer Science, 21(06):905-924, 2010.

[17] P. Keevash. Hypergraph Turan problems. In R. Chapman, editor, Surveys in Combinatorics 2011, volume 392 of London Mathematical Society Lecture Note Series, pages 83-140. Cambridge University Press, 2011.

[18] P. Keevash and B. Sudakov. The Turan number of the Fano plane. Combinatorica, 25(5):561-574, 2005.

[19] M. Krivelevich, R. Nathaniel, and B. Sudakov. Approximating coloring and maximum independent sets in 3-uniform hypergraphs. J. Algorithms, 41(1):99-113, 2001.

[20] L. Lovász. Coverings and colorings of hypergraphs. Proceedings of the Fourth Southeastern Conference on Combinatorics, Graph Theory, and Computing, pages 3-12, 1973.

[21] Y. Person and M. Schacht. An expected polynomial time algorithm for coloring 2-colorable 3graphs. Discrete Mathematics \& Theoretical Computer Science, 13(2):1-18, 2011.

[22] O. Pikhurko. Perfect matchings and $K_{4}^{3}$-tilings in hypergraphs of large codegree. Graphs and Combinatorics, 24(4):391-404, 2008.

[23] J. Radhakrishnan and A. Srinivasan. Improved bounds and algorithms for hypergraph 2-coloring. Random Structures \& Algorithms, 16:4-32, 2000.

[24] A. Razborov. On the minimal density of triangles in graphs. Combinatorics, Probability and Computing, 17(04):603-618, 2008.

[25] V. Rödl, A. Ruciński, and E. Szemerédi. A Dirac-type theorem for 3-uniform hypergraphs. Combinatorics Probability and Computing, 15(1-2):229-251, 2006.

[26] V. Rödl, A. Ruciński, and E. Szemerédi. Perfect matchings in large uniform hypergraphs with large minimum collective degree. J. of Combinatorial Theory A, 116(3):613-636, 2009.

[27] E. Szymańska. The complexity of vertex coloring problems in uniform hypergraphs with high degree. In D. M. Thilikos, editor, Graph Theoretic Concepts in Computer Science, volume 6410 of Lecture Notes in Computer Science, pages 304-314. Springer Berlin Heidelberg, 2010.

[28] E. Szymańska. The complexity of almost perfect matchings and other packing problems in uniform hypergraphs with high codegree. European J. of Combinatorics, 34(3):632 - 646, 2013. 
